\title{
Photometer performance assessment in Kepler science data processing
}

\author{
Jie Li ${ }^{*}$, Christopher Allen ${ }^{b}$, Stephen T. Bryson ${ }^{c}$, Douglas A. Caldwell ${ }^{\mathrm{a}}$, Hema Chandrasekaran ${ }^{\mathrm{a}}$, \\ Bruce D. Clarke ${ }^{\mathrm{a}}$, Jay P. Gunter ${ }^{\mathrm{b}}$, Jon M. Jenkins ${ }^{\mathrm{a}}$, Todd C. Klaus ${ }^{\mathrm{b}}$, Elisa V. Quintana ${ }^{\mathrm{a}}$, \\ Peter Tenenbaum ${ }^{\mathrm{a}}$, Joseph D. Twicken ${ }^{\mathrm{a}}$, Bill Wohler ${ }^{\mathrm{b}}$, Hayley $\mathrm{Wu}^{\mathrm{a}}$ \\ ${ }^{a}$ SETI Institute/NASA Ames Research Center, M/S 244-30, Moffett Field, CA, USA 94305; \\ borbital Sciences Corporation/NASA Ames Research Center, M/S 244-30, Moffett Field, CA, \\ USA 94305; \\ 'NASA Ames Research Center, M/S 244-30, Moffett Field, CA, USA 94305
}

\begin{abstract}
This paper describes the algorithms of the Photometer Performance Assessment (PPA) software component in the science data processing pipeline of the Kepler mission. The PPA performs two tasks: One is to analyze the health and performance of the Kepler photometer based on the long cadence science data down-linked via Ka band approximately every 30 days. The second is to determine the attitude of the Kepler spacecraft with high precision at each long cadence. The PPA component is demonstrated to work effectively with the Kepler flight data.
\end{abstract}

Keywords: Kepler mission, photometer performance assessment, instrument metric, attitude determination

\section{INTRODUCTION}

The Kepler mission is the first NASA mission capable of detecting Earth-size planets in the habitable zone of solar-like stars $^{1,2}$. The mission consists of a spacecraft with a single scientific instrument, a photometer, which is used to continuously and simultaneously measure the brightness variation of target stars to look for sequences of planetary transits. The Kepler spacecraft was launched into an Earth-trailing heliocentric orbit from Cape Canaveral, Florida on March 6, 2009. The baseline mission is planned for 3.5 years of operation following a 65-day commissioning period.

\subsection{Photometer of the Kepler spacecraft}

The photometer consists of a classic Schmidt telescope design with a $95 \mathrm{~cm}$ aperture and a field-of-view (FOV) of $\sim 115$ square degrees. The focal plane array consists of 42 Charge-Coupled Devices (CCDs). Each CCD has 1024×2200 pixels and two outputs with the serial channel on the long edge. Two CCDs are mounted together with a field flattener lens to form a nearly square module. As a result, there are a total of 21 modules, 84 outputs, and 96 megapixels on the Kepler photometer focal plane.

Each CCD integrates for 6 seconds and is read out in 0.5 seconds. The data from each pixel are co-added onboard the Kepler spacecraft for 30 minutes. Each of these 30-minute intervals is known as a long cadence, and the 30-minute coadded data as long cadence data. Using an onboard target table, data from the pixels of interest for each of $\sim 156,000$ target stars, along with collateral data used for calibration are extracted, compressed, and stored onboard.

During scientific observation of the entire mission, the attitude of the Kepler spacecraft is three-axis stabilized to keep the photometer FOV at a fixed region of the sky. The downsampled data of a sparse set of selected targets, which are used to monitor the photometer's health and status, are downloaded to the ground semi-weekly with an omni-directional $\mathrm{X}$-band $(8 \mathrm{GHz})$ antenna, which avoids interruption of scientific observation. Approximately every 30 days, scientific observation is halted and the spacecraft is maneuvered to point a Ka-band (32 GHz) high-gain antenna toward the Earth to download all the data in storage to the ground. In order to maintain the solar array toward the Sun, the spacecraft is rotated 90 degrees about the optical axis of the photometer every three months. The CCD layout on the focal plane is designed to be four-fold symmetric and CCD orientations are set up so that the readout row and column directions remain the same with each rotation (except for the center module).

*jie.li-1@nasa.gov; phone 1650 604-2458; fax 1650 604-2478 


\subsection{Photometer performance assessment software component}

Photometer Performance Assessment (PPA) is one of the software components of the science data processing pipeline of the Kepler mission ${ }^{3,4}$. One task of PPA is to analyze the health and performance of the Kepler photometer. The instrument metrics, either determined within the PPA component or provided as the outputs of the Calibration (CAL) ${ }^{5}$ or Photometric Analysis (PA) ${ }^{6}$ components, are analyzed with a track and trend algorithm where the mean and uncertainty are determined, out-of-bound values are identified and the trend is predicted. Another task of PPA is to determine the attitude of the Kepler spacecraft from the measured centroid position of the images of bright target stars. To compress the data to be processed, two-dimensional motion polynomials are built in the PA component to map the right ascension and declination of a target star to the row and column of the centroid of the star image for each output at each long cadence. The motion polynomials for all outputs are provided as inputs to PPA, which uses them to determine the attitude with high precision.

Compared to the Photometer Data Quality (PDQ) component ${ }^{7}$ which generates a report of the photometer's health and status from a subset of data downloaded via X-band semi-weekly, the PPA component analyzes the photometer's health and performance and determines the spacecraft's attitude from all the long cadence data downloaded via Ka-band every 30 days.

This paper is organized as follows: First, the Kepler instrument metrics are discussed, and the track and trend algorithm and the photometer performance report of the Kepler flight data are presented; Second, the data compression and smoothing with motion polynomials are described, the attitude determination algorithm with nonlinear least squares fit is described, and the attitude solutions of the Kepler flight data are demonstrated; And last, conclusions are presented.

\section{PHOTOMETER PERFORMANCE ANALYSIS}

\subsection{Kepler instrument metrics}

In the Kepler science data processing pipeline, data are processed per output ${ }^{8,9}$. In the PPA component the health and performance of the Kepler photometer are assessed by analyzing metric time series such as general instrument metrics, cosmic ray metrics, Combined Differential Photometric Precision (CDPP) metrics, etc. for each of 84 outputs and two additional metric time series - theoretical and achieved compression efficiencies for the full focal plane.

Table 1 lists general instrument metrics for each output and provides the unit and a brief description for each metric. The software component, in which each metric is determined, is shown in parenthesis in the description. For example, the metrics of black level, smear level and dark current are determined in $\mathrm{CAL}^{5}$ and the metrics of brightness and encircled energy are determined in $\mathrm{PA}^{6}$.

The background level is one of the general instrument metrics determined in PPA. The background values of an $11 \times 11$ grid of pseudo targets are determined for each output by evaluating the background polynomial provided by PA. The background level metric of the output is calculated as the weighted mean of the background values of the pseudo targets, where the weight is inversely proportional to the square of the uncertainty of each value. Figure 1 shows the background level metrics across the focal plane of the flight data on the Modified Julian Day (MJD) 55023, or July 11, 2009. The background level increases gradually from the lower left to the upper right corner of the figure, since the upper right corner is closer to the galaxy equator (where more dim stars are located), as a result of the spacecraft's quarterly roll.

In PPA, theoretical and achieved compression efficiencies of the full focal plane are determined and analyzed as metrics of efficiency of the onboard data compression algorithm, as well as metrics of the margin of the memory usage of the onboard computer. Compression efficiencies of the full focal plane are calculated from the corresponding metrics of each output, provided by the CAL component, with the following equation:

$$
C E_{F F P}=\frac{\sum_{i=1}^{84} s_{i} \cdot C E_{i}}{\sum_{i=1}^{84} s_{i}}
$$


where $s_{i}$ is the number of code symbols of output $i(i=1, \ldots 84), C E_{i}$ is the theoretical or achieved compression efficiency of the output, and $C E_{F F P}$ is the corresponding compression efficiency of the full focal plane.

Table 1. General instrument metrics for each of 84 outputs.

\begin{tabular}{|c|c|c|}
\hline Metrics & Unit & Description \\
\hline black level & ADU/read & Median of black level (CAL) \\
\hline smear level & electron/cadence & Median of smear level (CAL) \\
\hline dark current & electron/second & Median of dark current (CAL) \\
\hline brightness & dimensionless & $\begin{array}{l}\text { Mean of the ratio of received flux to the expected flux of the target star } \\
\text { (PA) }\end{array}$ \\
\hline encircled energy & pixel & $\begin{array}{l}\text { Mean of the radius of the circle to hold } 95 \% \text { of the energy of the target } \\
\text { star (PA) }\end{array}$ \\
\hline background level & electron/cadence & Median of background level (PPA) \\
\hline centroid mean row & pixel & $\begin{array}{l}\text { Mean of difference in the row between the centroid of the image of the } \\
\text { target star and its predicted position from nominal attitude (PPA) }\end{array}$ \\
\hline centroid mean column & pixel & $\begin{array}{l}\text { Mean of difference in the column between the centroid of the image of } \\
\text { the target star and its predicted position from nominal attitude (PPA) }\end{array}$ \\
\hline plate scale & arcsecond/pixel & $\begin{array}{l}\text { Mean of the ratio of angular distance between stars and the linear } \\
\text { distance between their images on the focal plane (PPA) }\end{array}$ \\
\hline $\begin{array}{l}\text { theoretical } \\
\text { compression efficiency }\end{array}$ & bit/pixel/cadence & $\begin{array}{l}\text { Theoretical compression efficiency of the onboard data compression } \\
\text { algorithm (CAL) }\end{array}$ \\
\hline $\begin{array}{l}\text { achieved } \\
\text { compression efficiency }\end{array}$ & bit/pixel/cadence & $\begin{array}{l}\text { Actual achieved compression efficiency of the onboard data } \\
\text { compression algorithm (CAL) }\end{array}$ \\
\hline
\end{tabular}

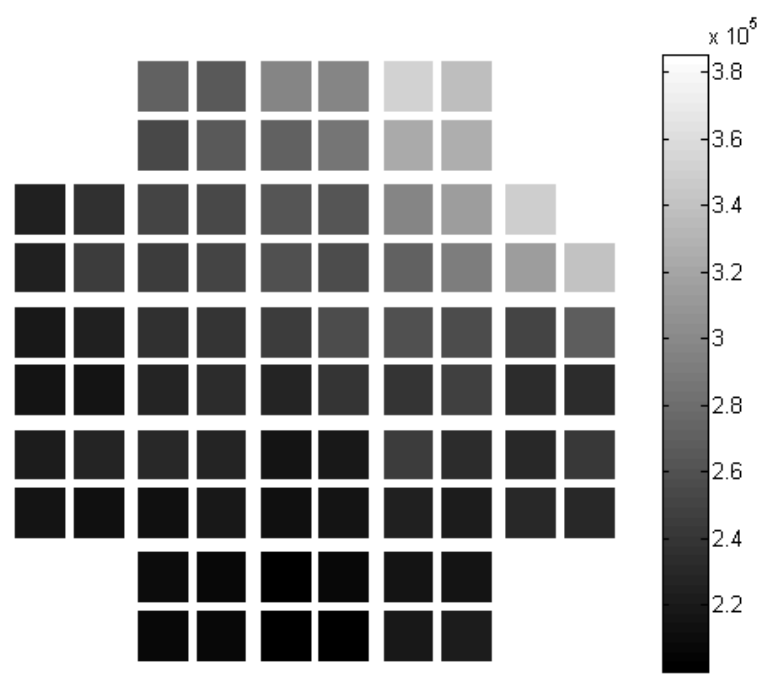

Figure 1. Background level across the focal plane of the flight data for MJD 55023 (unit: electron/cadence). 
Figure 2 shows achieved compression efficiency of the full focal plane of flight data for second quarter of Kepler science data collection, ranging from MJD 55002 (June 20, 2009) to MJD 55091 (September 17, 2009). Spikes are observed in the achieved compression efficiency after events of attitude variations such as a safe mode, an attitude maneuver, or an attitude tweak that lead to a change in the star images. As a result, redundancy of raw data decreases and size of compressed data increases.



Figure 2. Achieved compression efficiency of the full focal plane for second quarter flight data

Cosmic ray metrics are a set of time series for the hit rate of cosmic ray and the mean and variance of the distribution of cosmic ray energy for a specified group of pixels on the CCD. For each output, CAL generates cosmic ray metrics of black pixels, masked smear pixels, and virtual smear pixels ${ }^{5}$, and PA generates cosmic ray metrics of target star pixels and background pixels ${ }^{6}$.

CDPP is a metric for the effective white noise standard deviation in a time interval for the flux time series of the target stars. It determines the signal-to-noise ratio of the transit with a given depth and duration of that interval. In PPA, target stars are grouped in seven bins according to their magnitude, from Magnitude 9 to Magnitude 15, for each output. The expected and measured CDPP metrics are determined for each magnitude bin from the flux value and uncertainty time series, generated by the Pre-search Data Conditioning (PDC) component $^{10}$, and the CDPP time series, generated by the Transiting Planet Search (TPS) component ${ }^{11}$, respectively.

\subsection{Track and trend algorithm}

The time series of the instrument metrics are analyzed with the track and trend algorithm, which identifies metric samples beyond the fixed/adaptive upper and lower bounds, and predicts whether the metric crosses the fixed/adaptive bounds within a horizon time beyond the end of the metric time series. Note that the fixed upper and lower bounds of the metrics and the horizon time for prediction are pre-defined input parameters of PPA.

Adaptive upper and lower bounds are determined from estimates of time-varying mean and noise/variation levels of metric time series. For a given metric time series $\left\{d_{k}, k=1,2, \ldots\right\}$, the mean value, variance and uncertainty are estimated with a first order lowpass filtering algorithm as follows:

$$
\begin{aligned}
& m_{k}=\left(1-\beta_{m}\right) m_{k-1}+\beta_{m} d_{k} \\
& v_{k}=\left(1-\beta_{v}\right) v_{k-1}+\beta_{v}\left(d_{k}-m_{i}\right)^{2} \\
& u_{k}=\sqrt{v_{k}}
\end{aligned}
$$


where $d_{k}$ is the metric sample at the $k$-th long cadence, $m_{k}, v_{k}$ and $u_{k}$ are the estimated mean value, variance and uncertainty at the same long cadence respectively. $\beta_{m}$ and $\beta_{v}$, ranging between 0 and 1 , are filtering coefficients for the mean value and variance respectively. A filtering coefficient closer to 0 suppresses the noise more effectively at the cost of sluggishness to follow the time variation of the metric time series. Based on the estimates, the adaptive upper and lower bounds are defined by the time series $\left\{m_{k}+u_{k}\right\}$ and $\left\{m_{k}-u_{k}\right\}$ respectively.

The trend of metric time series is determined by selecting metric samples in a pre-defined interval (for example, five days) at the end of the time series and then fitting the selected data with a linear model. It is straight-forward to determine when the trend line specified by the linear model crosses the fixed and adaptive bounds.

An alert is generated, attached with an alert level, when a metric sample is identified to be beyond fixed or adaptive bounds or when there is a bound-crossing event within the horizon time beyond the end of a metric time series. For example, samples beyond the fixed and adaptive bounds are marked red and yellow respectively, and the alert level is marked green when no samples are identified beyond the fixed or adaptive bounds,

Figure 3 shows a diagram of the track and trend algorithm and Figure 4 illustrates the track and trend of achieved compression efficiency of the full focal plane for second quarter flight data. In Figure 4, the metric samples are plotted with a solid line and dot markers, the adaptive upper and lower bounds are plotted with dash lines, and the trend is plotted with a dash-dot line. The estimated uncertainty increases to indicate higher noise/variation levels when the spiky metric samples are encountered, and the band defined by the adaptive upper and lower bounds becomes wider accordingly. When the estimated uncertainty converges to the normal noise/variation level, the band shrinks, showing adaptation to the time-varying feature of the mean and noise variation level of the metric time series.

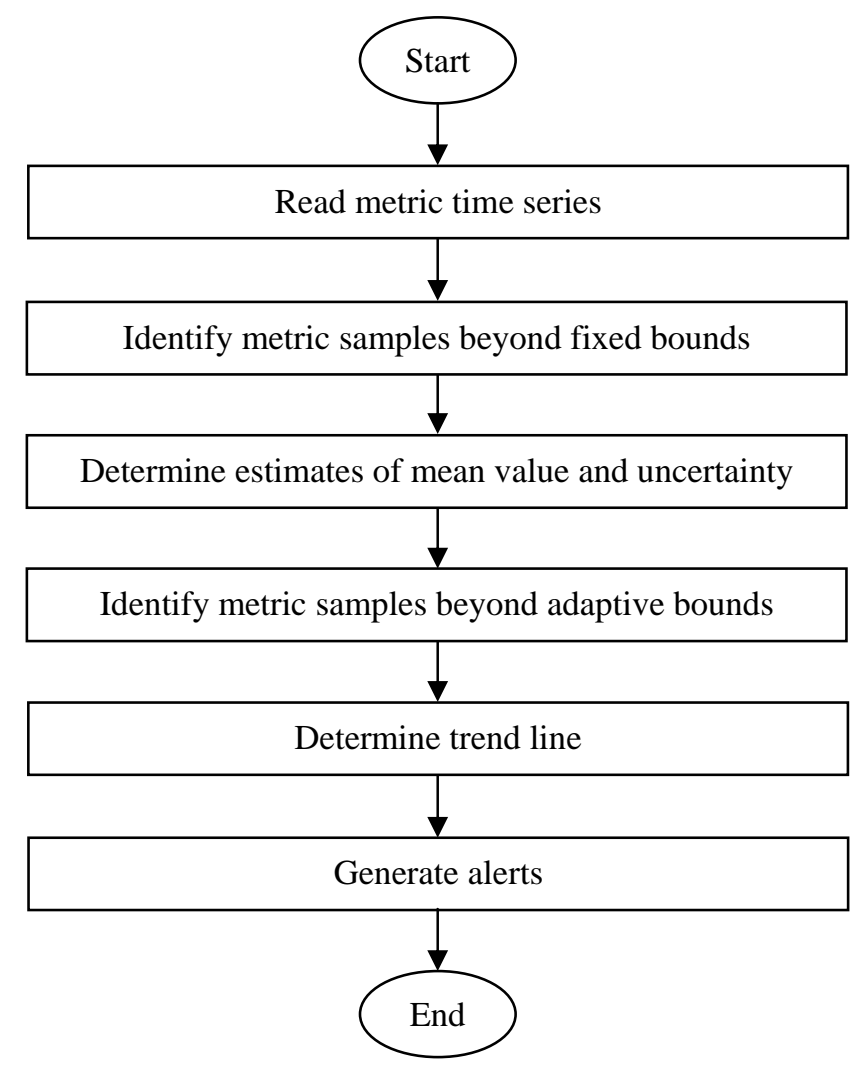

Figure 3. The track and trend algorithm of instrument metrics. 


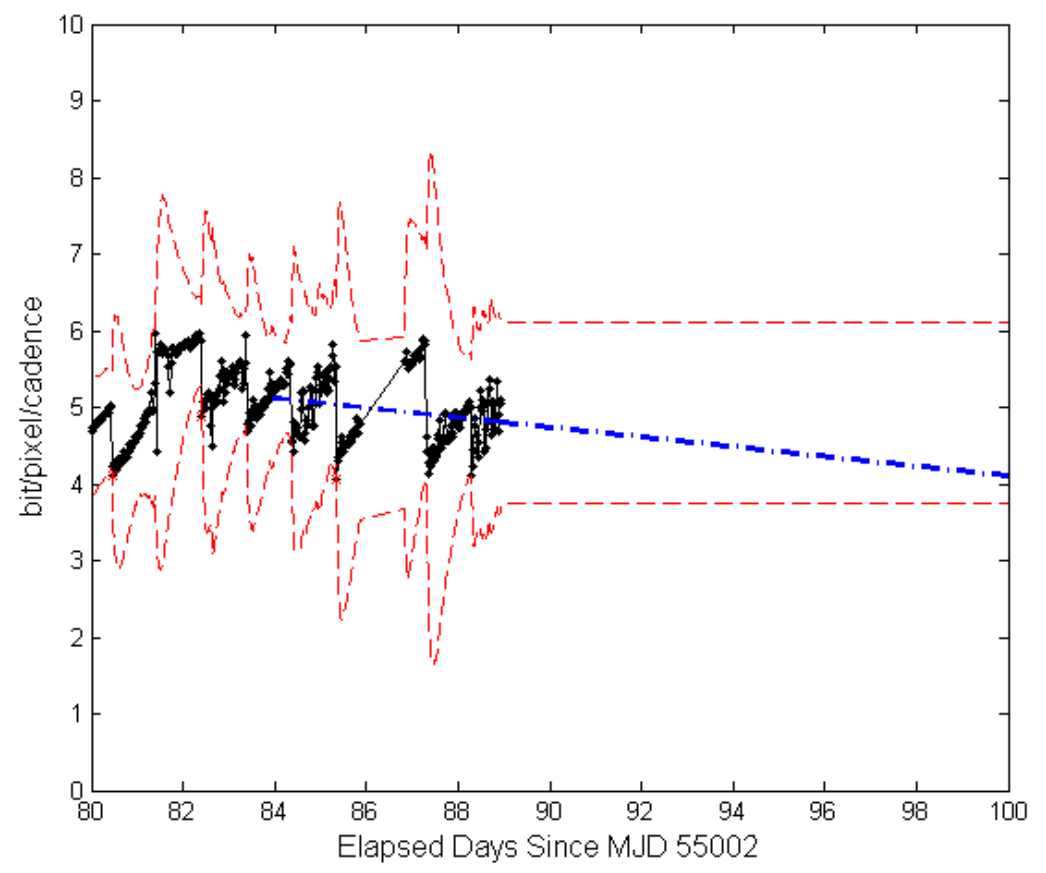

Figure 4. Track and trend of achieved compression efficiency of the full focal plane for second quarter flight data

\subsection{Photometer performance reports of flight data}

Photometer performance reports are generated by PPA for each output and for the full focal plane. Figures 5a and 5b show the general instrument metrics dashboard of second quarter flight data for module 6/output 3 and the full focal plane respectively. As seen in Figure 5a, each of the general instrument metrics corresponds to a rectangle color-coded with the track alert level. Figure $5 \mathrm{~b}$ is an aggregation of the dashboard of all 84 outputs on the focal plane, where module 6/output 3, is the first output of the third row. Each small rectangle in the square of an output corresponds to a metric, as labeled in Figure 5a. The two squares in the upper right corner of Figure 5b correspond to the metrics of theoretical and achieved compression efficiencies of the full focal plane. It is seen that the track alert levels of most metrics for the outputs across the focal plane are green, demonstrating that the Kepler photometer is in good health and performs well in the second quarter. The red alert level of achieved compression efficiency of some outputs is due to the spiky samples following the attitude variations, as shown in Figure 2. The in-flight performance of the Kepler instrument is discussed in detail by Caldwell, D.A., et al. ${ }^{12,13}$

\section{ATTITUDE DETERMINATION WITH HIGH PRECISION}

\subsection{Measurement data compression and smoothing}

In PPA, the attitude of the Kepler spacecraft is determined from the measured centroid position of the images of bright target stars. Approximately 200 bright target stars are selected for each output. Based on the measurements of $\sim 16,000$ star images across the focal plane, the attitude solution of the Kepler spacecraft is determined with high precision at each 30-minute long cadence. However, the size of the measurement data for a month or a quarter, which is the length of data processed by the Kepler science data processing pipeline, is so large that it is difficult to process the data directly to determine the attitude. As a solution to the problem, two motion polynomials, one for row and one for column, are generated in the PA component for each output and each long cadence. By fitting the measured rows or columns of the centroids of the images of 200 bright target stars from an output into a two-dimensional polynomial of the corresponding right ascensions and declinations of the stars, the motion polynomial builds an empirical mapping from the position of a target in the sky to the measured position of the target on the focal plane of the Kepler photometer. 
(a)

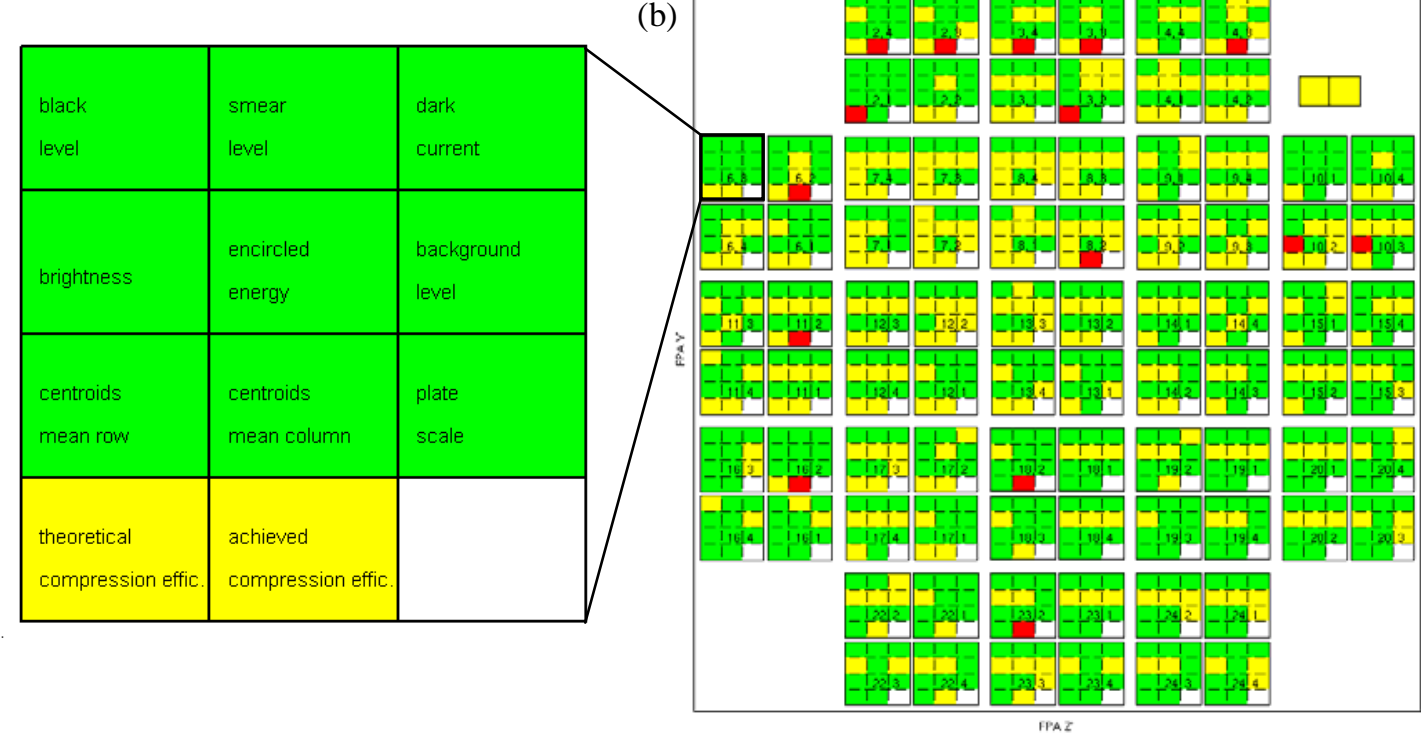

Figure 5. General instrument metrics dashboard for second quarter flight data. (a) module 6/output 3; (b) full focal plane.

In PPA, a set of pseudo stars is selected for each output first, and the positions of the selected pseudo stars in the sky are determined; then, the "measured" rows and columns of the pseudo stars, together with the covariance matrices, which are calculated from the row and column motion polynomials, are used as input measurements for the attitude determination. Considering the order of the motion polynomials is limited (usually $<4$ ), the size of the "measurement" data of the pseudo stars, which is input to the attitude determination function in PPA, is reduced significantly and the noise in the measurements of original target stars is suppressed effectively as well.

For each output, pseudo stars are selected from a $3 \times 3$ grid. The requirement that input measurements should be uncorrelated imposes constraints on the selection of pseudo stars. The correlation of rows or columns of a set of pseudo stars, determined by evaluating the two-dimensional motion polynomial, is related to the order of the motion polynomial and the geometry of the pseudo stars. Figure 6 illustrates the selection of pseudo stars of an output from a $3 \times 3$ grid in case of (a) $N_{m p}=0$, (b) $N_{m p}=1$, (c) $N_{m p}=2$, and (d) $N_{m p}>=3$ respectively, where $N_{m p}$ is the order of the motion polynomial.

The selected pseudo stars for each of 84 outputs are fixed in the sky. Their positions, in right ascension and declination, are determined from the nominal attitude of the Kepler spacecraft, which will be discussed later.

\subsection{Attitude determination with nonlinear least squares fit}

The attitude of the Kepler spacecraft is described by the right ascension and declination of the optical axis of the photometer, denoted as $\alpha_{S}$ and $\delta_{S}$ respectively, and a rotation angle along the optical axis, denoted as $\phi_{S}$. A 3-2-1 transformation with Euler angles of $\alpha_{S},-\delta_{S}$ and $\phi_{S}$ rotates the inertial equatorial coordinate system, with the $\mathrm{X}$ axis pointing to the vernal equinox, to the spacecraft body fixed photometer focal plane coordinate system; thus, pointing the photometer FOV to a fixed region of the sky. The nominal values of $\alpha_{S}$ and $\delta_{S}$ are $290.66667^{\circ}$ and $44.5^{\circ}$ for the Kepler science mode, which are constant for the entire mission. The rotation angle $\phi_{S}$ varies by multiples of $90^{\circ}$ corresponding to quarterly spacecraft roll maneuvers, with a nominal value of $123^{\circ}$ in the quarter from June 20, 2009 (MJD 55002) to September 17, 2009 (MJD 55091). 


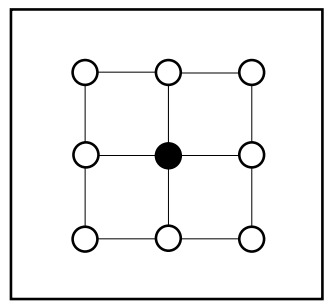

(a)

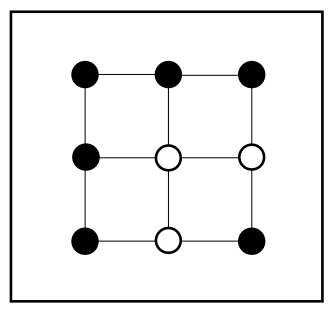

(c)

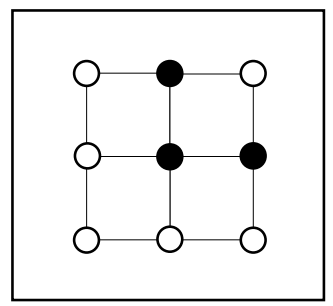

(b)



(d)

Figure 6. Selection of pseudo stars of an output from a $3 \times 3$ grid in case of (a) $N_{m p}=0$, (b) $N_{m p}=1$, (c) $N_{m p}=2$, and (d) $N_{m p}>=3$ respectively, where $N_{m p}$ is the order of the motion polynomial.

Let $\alpha_{i, j}$ and $\delta_{i, j}$ denote the right ascension and declination of the $j$-th selected pseudo star of output $i$, where $i=1, \ldots$ $84, j=1, \ldots, J_{i}$. $J_{i}$ is the total number of selected pseudo stars of output $i$. As discussed in Section 3.1, the "measured" row and column of the centroid of the image of the pseudo star at the $k$-th long cadence, denoted as $r_{i, j, k}$ and $c_{i, j, k}$ respectively, are determined by evaluating the row and column motion polynomials at $\alpha_{i, j}$ and $\delta_{i, j}$, as expressed in the equations below:

$$
\begin{aligned}
& r_{i, j, k}=M P_{\text {row }, i, k}\left(\alpha_{i, j}, \delta_{i, j}\right) \\
& c_{i, j, k}=M P_{c o l, i, k}\left(\alpha_{i, j}, \delta_{i, j}\right)
\end{aligned}
$$

where $M P_{r o w, i, k}(\cdot, \cdot)$ and $M P_{c o l, i, k}(\cdot, \cdot)$ are two-dimensional motion polynomials of the row and column for output $i$ at the $k$-th long cadence. The covariance matrices of the measurements $r_{i, j, k}$ and $c_{i, j, k}\left(j=1, \ldots J_{i}\right)$, denoted as $W_{\text {row }, i, k}$ and $W_{c o l, i, k}$, are determined from the covariance matrices of the coefficients of $M P_{r o w, i, k}(\cdot, \cdot)$ and $M P_{c o l, i, k}(\cdot, \cdot)$.

The "predicted" row and column of the centroid of the image of the pseudo star at the $k$-th long cadence, denoted as $\hat{r}_{i, j, k}$ and $\hat{c}_{i, j, k}$ respectively, are functions of the right ascension $\alpha_{i, j}$ and declination $\delta_{i, j}$ of the pseudo star, and the attitude parameters at the $k$-th long cadence, denoted as $\alpha_{S, k}, \delta_{S, k}$ and $\phi_{S, k}$, as following:

$$
\begin{aligned}
& \hat{r}_{i, j, k}=M D_{\text {row }, i}\left(\alpha_{i, j}, \delta_{i, j}, \alpha_{S, k}, \delta_{S, k}, \phi_{S, k}\right) \\
& \hat{c}_{i, j, k}=M D_{c o l, i}\left(\alpha_{i, j}, \delta_{i, j}, \alpha_{S, k}, \delta_{S, k}, \phi_{S, k}\right)
\end{aligned}
$$

where $M D_{r o w, i}$ and $M D_{c o l, i}$ denote the models of the spacecraft attitude and the photometer optics, including models of stellar velocity aberration, photometer optical distortion and the focal plane geometry etc. ${ }^{14,15}$. 
The attitude solution at the $k$-th long cadence is determined as the attitude parameters $\alpha_{S, k}$, $\delta_{S, k}$ and $\phi_{S, k}$ which minimize the nonlinear least squares function as follows:

$$
\begin{aligned}
& J\left(\alpha_{S, k}, \delta_{S, k}, \phi_{S, k}\right)= \\
& \sum_{i=1}^{84}\left[\left(V_{\text {row }, i, k}-\hat{V}_{\text {row }, i, k}\right)^{T} W_{\text {row }, i, k}^{-1}\left(V_{\text {row }, i, k}-\hat{V}_{\text {row }, i, k}\right)+\left(V_{c o l, i, k}-\hat{V}_{c o l, i, k}\right)^{T} W_{c o l, i, k}^{-1}\left(V_{c o l, i, k}-\hat{V}_{c o l, i, k}\right)\right]
\end{aligned}
$$

where

$$
\begin{aligned}
& V_{\text {row }, i, k}=\left[\begin{array}{llll}
r_{i, 1, k} & r_{i, 2, k} & \ldots & r_{i, J i, k}
\end{array}\right]^{T} \\
& V_{\text {col }, i, k}=\left[\begin{array}{llll}
c_{i, 1, k} & c_{i, 2, k} & \ldots & c_{i, J i, k}
\end{array}\right]^{T} \\
& \hat{V}_{\text {row }, i, k}=\left[\begin{array}{llll}
\hat{r}_{i, 1, k} & \hat{r}_{i, 2, k} & \ldots & \hat{r}_{i, J i, k}
\end{array}\right]^{T} \\
& \hat{V}_{\text {col }, i, k}=\left[\begin{array}{llll}
\hat{c}_{i, 1, k} & \hat{c}_{i, 2, k} & \ldots & \hat{c}_{i, J i, k}
\end{array}\right]^{T}
\end{aligned}
$$

Figure 7 shows a diagram of the attitude determination algorithm. As depicted in the diagram, the attitude determination algorithm is implemented in the following steps. First, a set of pseudo target stars is selected for each output based on the orders of the corresponding motion polynomials. The right ascension and declination of the pseudo star are determined from the nominal attitude of the Kepler spacecraft. Second, the "measured" rows and columns of the centroids of the pseudo stars of each of the 84 outputs at a 30-minute long cadence are calculated by evaluating the corresponding row and column motion polynomials, as shown in Equation (3). The covariance matrices of the measurements of the row and column are determined as well from the covariance matrices of the coefficients of the motion polynomials. Then, the outliers in the "measured" rows and columns are identified and removed with robust regression, and the attitude solution is determined at the long cadence as the nonlinear least-squares fit of the cleaned "measured" data of the pseudo stars across the focal plane, as shown in Equation (5). Finally, a metric of the maximum position offset of star images on the focal plane between the attitude solution and the nominal attitude is determined to see how well images of target stars are located within corresponding apertures defined by the target table.

\subsection{Attitude solutions of flight data}

Figure 8 shows the difference in declination between the nominal attitude and the attitude solution for second quarter flight data. The timestamps of the figure are elapsed days referenced to MJD 55002.0, or 0h0m0s June 20, 2009. Several events of the Kepler spacecraft are observed in this figure. The spacecraft goes into safe mode on day 12 and comes back to normal science orientation on day 14. A transient in the attitude is observed following the safe mode, mainly due to temperature variations. On days 54 and 86, the spacecraft goes into coarse pointing mode. During days 61 and 62, the spacecraft is maneuvered to point its high-gain Ka-band antenna toward the earth and science data is downloaded to the ground. A pointing drift is observed in the measured declination relative to the nominal science attitude ${ }^{16}$. In order to keep all selected target stars within the corresponding apertures defined by the target table, the drift is corrected by attitude tweaks on days 0,31 , and 77. As expected, jumps in the spacecraft attitude parameters are observed during the attitude maneuver and attitude tweak. Note that the attitude variations, as shown in Figure 8, result in systematic errors in the flux time series of target stars, which need to be modeled and removed by the PDC component ${ }^{10}$ before the search for planets is initiated. 


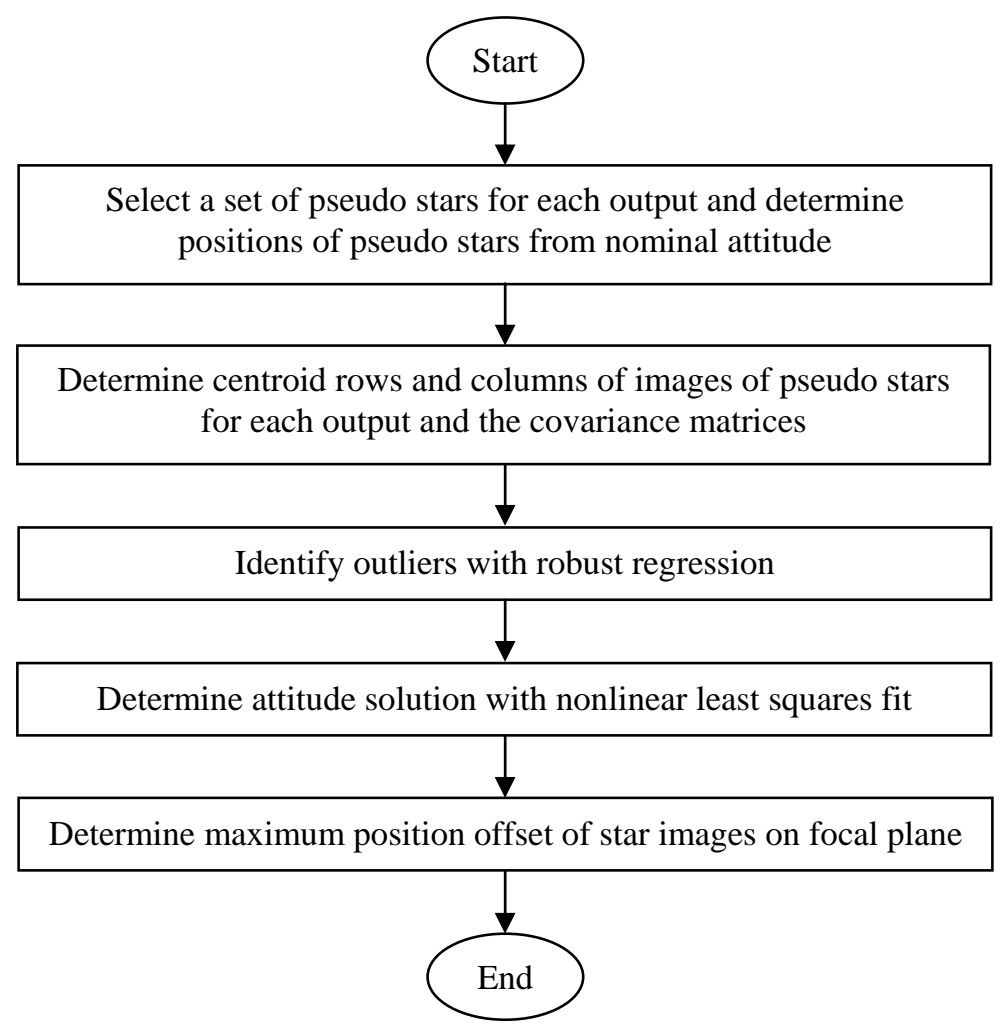

Figure 7. The attitude determination algorithm.

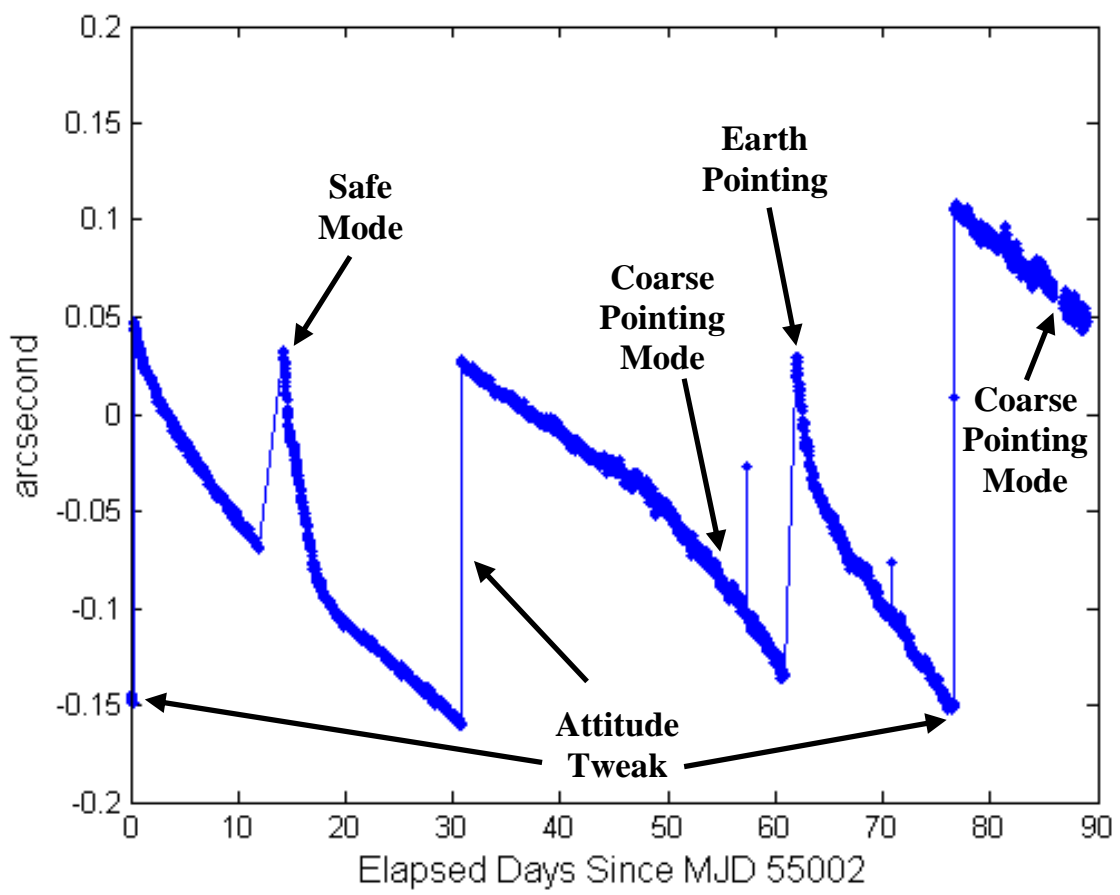

Figure 8. Difference in declination between nominal attitude and attitude solution for second quarter flight data 


\section{CONCLUSIONS}

This paper discusses the algorithms of the PPA software component in the science data processing of the Kepler mission. The health and performance of the Kepler photometer are assessed by analyzing time series of instrument metrics with the track and trend algorithm, and photometer performance reports are generated for each of 84 outputs and the full focal plane. With the motion polynomials for each output and each long cadence, the attitude of the Kepler spacecraft is determined with high precision at each long cadence from the compressed and smoothed measurements of the centroids of images of 16,000 bright target stars across the focal plane. The PPA component is demonstrated to work effectively with the Kepler flight data.

Funding for the Kepler mission is provided by NASA’s Discovery Program Office, Science Mission Directorate.

\section{REFERENCES}

[1] Borucki, W.J., et al., “Kepler planet-detection mission: introduction and first results”, Science, 327(5968), 977-980 (2010).

[2] Koch, D.G., et al., “Kepler Mission design, realized photometric performance, and early science”, Astrophysical Journal Letters, 713(2), L79-L86 (2010).

[3] Jenkins, J.M., et al., "Overview of the Kepler science processing pipeline”, Astrophysical Journal Letters, 713(2), L87-L91 (2010).

[4] Middour, C., et al., "Kepler Science Operations Center architecture”, Proc. SPIE, this volume (2010).

[5] Quintana, E.V., et al., "Pixel-level calibration in the Kepler Science Operations Center pipeline", Proc. SPIE, this volume (2010).

[6] Twicken, J.D., et al., "Photometric analysis in the Kepler Science Operations Center pipeline”, Proc. SPIE, this volume (2010).

[7] Chandrasekaran, H., et al., "Semi-weekly monitoring of the performance and attitude of Kepler using a sparse set of targets”, Proc. SPIE, this volume (2010).

[8] Klaus, T.C., et al., "The Kepler Science Operations Center science pipeline configuration and execution”, Proc. SPIE, this volume (2010).

[9] Hall, J.R., et al., "Kepler science operations processes, procedures, and tools”, Proc. SPIE, 7737, in press (2010).

[10] Twicken, J.D., et al., "Presearch data conditioning in the Kepler Science Operations Center", Proc. SPIE, this volume (2010).

[11] Jenkins, J.M., et al., "Transiting planet search in the Kepler pipeline”, Proc. SPIE, this volume (2010).

[12] Caldwell, D.A., et al., "Instrument performance in Kepler’s first months”, Astrophysical Journal Letters, 713(2), L92-L96 (2010).

[13] Caldwell, D.A., et al., “Kepler instrument performance: an in-flight update”, Proc. SPIE, 7731, in press (2010).

[14] Allen, C., Klaus T.C. and Jenkins, J.M., "Kepler Mission’s focal plane characterization models implementation”, Proc. SPIE, this volume (2010).

[15] Tenenbaum, P. and Jenkins, J.M., “Focal plane geometry characterization of the Kepler Mission”, Proc. SPIE, this volume (2010).

[16] Haas, M.R., et al., “Kepler science operations”, Astrophysical Journal Letters, 713(2), L115-L119 (2010). 